\title{
Instabilités de contact : analyses temporelle, aux valeurs propres et validation expérimentale
}

\author{
Anissa Meziane $^{1, a}$, Laurent Baillet ${ }^{2}$, Bernard Laulagnet $^{3}$, Claude Godeau $^{1}$ \\ ET Yves Berthier ${ }^{1}$ \\ 1 Laboratoire de Mécanique des Contacts et des Solides, INSA-Lyon, CNRS UMR 5259, 69621 Villeurbanne, France \\ 2 Laboratoire de Géophysique Interne et de Tectonophysique, Université Joseph Fourier CNRS, BP 53, \\ 38041 Grenoble Cedex 9, France \\ 3 Laboratoire de Vibrations et Acoustique, INSA-Lyon, 20 avenue Albert Einstein, 69621 Villeurbanne, France
}

Reçu le 8 mars 2007, accepté le 18 septembre 2007

\begin{abstract}
Résumé - Les instabilités générées par frottement sont responsables des divers bruits tels que le crissement, le sifflement ou le broutement... Pour modéliser et comprendre ce phénomène d'instabilités, les analyses temporelles et aux valeurs propres sont utilisées sur un système modèle constitué de deux poutres en contact. Dans l'analyse aux valeurs propres, l'instabilité se manifeste par la coalescence de deux modes propres du système. Dans l'analyse temporelle, l'instabilité est caractérisée par des zones d'adhérence ou de décollement qui apparaissent au niveau de la surface de contact. Les résultats issus des deux analyses sont cohérents et complémentaires. Une validation expérimentale a été effectuée et montre une bonne corrélation entre les résultats numériques et expérimentaux.
\end{abstract}

Mots clés : Instabilités de contact / crissement / contact / dynamique / éléments-finis

\begin{abstract}
Friction-induced instabilities: modal, transient analysis and experimental validation. The vibrations generated at the interface between the two bodies in friction are responsible for various noises such as squealing, juddering, hammering, hooting, etc. In order to model and understand friction-induced vibration phenomenon, two types of analysis, modal analysis and transient analysis, are compared in this article. This study has been made on a simplified system composed of two beams in contact. In modal analysis, instabilities appear when a pair of modes merges. Eigenvalues with positive real parts are identified as potentially unstable modes. In transient analysis, one speaks about instabilities when stick or separation zones appear in the contact surfaces. Results have been compared and both analysis give coherent and complementary results. An experimental validation has been made and shows a good correlation between experimental and numerical results.
\end{abstract}

Key words: Friction-induced instabilities / squeal / contact / dynamics / Finite-elements

\section{Introduction}

Lorsque deux corps sont en contact frottant, des vibrations peuvent apparaître à l'interface. Ces vibrations induites par frottement (ou instabilités de contact) sont responsables de divers bruits et de concentration de contraintes. Dans l'application du freinage automobile, ces vibrations peuvent donner lieu à du broutement, du crissement, du bourdonnement... Un vaste panorama de

\footnotetext{
a Auteur pour correspondance :

anissa.meziane@insa-lyon.fr
}

la dynamique des vibrations induites par frottement est présenté par Ibrahim [1,2]. Akay [3] présente une vue d'ensemble de l'acoustique du frottement. Dans la littérature, les nombreuses études expérimentales, analytiques [4] et numériques montrent que le phénomène d'instabilité de contact est complexe et qu'il n'est pas encore totalement maîtrisé.

En 1938, Mills [5] montre que des instabilités de contact peuvent apparaître lorsque le coefficient de frottement décroît avec la vitesse relative. Block [6] complète cette théorie en montrant que ces vibrations instables peuvent également être obtenues lorsque le cofficient 


\section{Nomenclature}

\begin{tabular}{|c|c|}
\hline $\mathbf{C}$ & Matrice d'amortissement du système $\left(\mathbf{C}=\alpha_{1} \mathbf{M}+\alpha_{2} \mathbf{K}\right)\left(\mathrm{Ns.m}^{-1}\right)$ \\
\hline$F$ & Valeur absolue de la force appliquée sur la poutre $\Omega_{1}(\mathrm{~N})$ \\
\hline$f$ & Vecteur des forces extérieures nodales du système $(\mathrm{N})$ \\
\hline G & Matrice globale des conditions au niveau du contact \\
\hline $\mathbf{K}$ & Matrice de raideur du système $\left(\mathrm{N} . \mathrm{m}^{-1}\right)$ \\
\hline $\mathbf{K}^{\mathrm{c}}$ & Matrice de raideur du système avec contact $\left(\mathrm{N} . \mathrm{m}^{-1}\right)$ \\
\hline$K_{\mathrm{c}}$ & Raideur de contact (N.m $\left.{ }^{-1}\right)$ \\
\hline$\hat{L}$ & Longueur caractéristique de la loi de Prakash-Clifton (m) \\
\hline M & Matrice de masse du système (kg) \\
\hline$t, \Delta t$ & Temps (s), pas de temps (s) \\
\hline $\boldsymbol{u}, \dot{\boldsymbol{u}}, \ddot{u}$ & Vecteurs déplacement $(\mathrm{m})$, vitesse $\left(\mathrm{m} \cdot \mathrm{s}^{-1}\right)$ et accélération $\left(\mathrm{m} \cdot \mathrm{s}^{-2}\right)$ \\
\hline$V$ & Vitesse imposée à la poutre $2\left(\mathrm{~m} . \mathrm{s}^{-1}\right)$ \\
\hline$\hat{V}$ & Vitesse caractéristique de la loi de Prakash-Clifton $\left(\mathrm{m} . \mathrm{s}^{-1}\right)$ \\
\hline $\boldsymbol{x}$ & Vecteur des coordonnées du système $(\mathrm{m})$ \\
\hline$\alpha_{1}, \alpha_{2}$ & Paramètres d'amortissement proportionnel $\left(\mathrm{s}^{-1}, \mathrm{~s}\right)$ \\
\hline$\beta_{2}$ & Paramètre d'amortissement numérique \\
\hline$\varepsilon$ & Taux d'amortissement \\
\hline$\varphi$ & Valeur propre du système matriciel \\
\hline$\lambda$ & Vecteur des forces de contact $(\mathrm{N})$ \\
\hline$\mu$ & Coefficient de frottement de Coulomb \\
\hline$\omega$ & Pulsation propre (rad.s ${ }^{-1}$ ) \\
\hline$\theta$ & Angle relatif entre les deux poutres $\left(^{\circ}\right)$ \\
\hline$\hat{\tau}$ & Temps de réponse caractéristique de la loi de Prakash-Clifton (s) \\
\hline \multicolumn{2}{|c|}{ Indices } \\
\hline$n$ & Relatif à l'instant $t_{n}=n \Delta t$ \\
\hline$n+1$ & Relatif à l'instant $t_{n+1}=t_{n}+\Delta t$ \\
\hline$n-1$ & Relatif à l'instant $t_{n-1}=t_{n}-\Delta t$ \\
\hline$\Omega_{1}, \Omega_{2}$ & Relatif aux poutres $\Omega_{1}$ et $\Omega_{2}$ \\
\hline \multicolumn{2}{|c|}{ Exposant } \\
\hline$\vec{n}$ & Selon la normale \\
\hline$\vec{t}$ & Selon la tangentielle \\
\hline $\mathrm{T}$ & Transposée \\
\hline$*$ & Sans prise en compte des forces de contact \\
\hline $\mathrm{p}$ & État perturbé à partir de la position d'équilibre \\
\hline
\end{tabular}

de frottement statique est supérieur au coefficient de frottement dynamique. En 1961, Spurr [7] développe le modèle de «Sprag-Slip ». Il montre l'importance de la cinématique de contact en obtenant une condition d'instabilité sur l'angle d'attaque du contact avec un coefficient de frottement constant. En 1972, North [8] montre que les instabilités de contact sont dues à une coalescence de deux valeurs propres du système, une des deux valeurs propres possédant une partie réelle positive. Les instabilités de contact sont obtenues avec un coefficient de frottement constant et correspondent à des vibrations autoentretenues du système.

Par la suite, avec l'augmentation de la puissance de calcul, les études numériques ont fait leur apparition. Elles s'appuient sur la méthode des éléments-finis qui permet à une large gamme de systèmes (géométrie des corps en contact, conditions limites et modélisation du contact) d'être traitée. Ces études numériques sont essentiellement basées sur deux types d'analyse : les analyses aux valeurs propres et les analyses temporelles.
Le principe des analyses aux valeurs propres, linéaires, est de calculer les modes et valeurs propres du système couplé constitué par les deux corps en contact. L'instabilité, caractérisée par la coalescence de deux modes qui deviennent complexes, est appelée instabilité par flottement [9-14]. Cette analyse est peu coûteuse en terme de temps de calculs, mais ne permet pas de connaître le comportement du système pendant l'instabilité. En revanche, les analyses temporelles, qui prennent en compte le caractère non-linéaire du contact avec frottement, fournissent l'évolution des grandeurs mécaniques (contraintes, déformations) en fonction du temps, permettant ainsi de connaître la dynamique globale et locale (au niveau du contact) du système [15-18]. Pour les systèmes étudiés, les réponses en temps mettent en évidence des comportements instables harmoniques, quasi-harmoniques ou parfois chaotiques. L'inconvénient de cette analyse est un temps de calcul important qui rend les études paramétriques plus lourdes à mettre en œuvre qu'avec l'analyse aux valeurs propres. Il existe d'autres techniques 


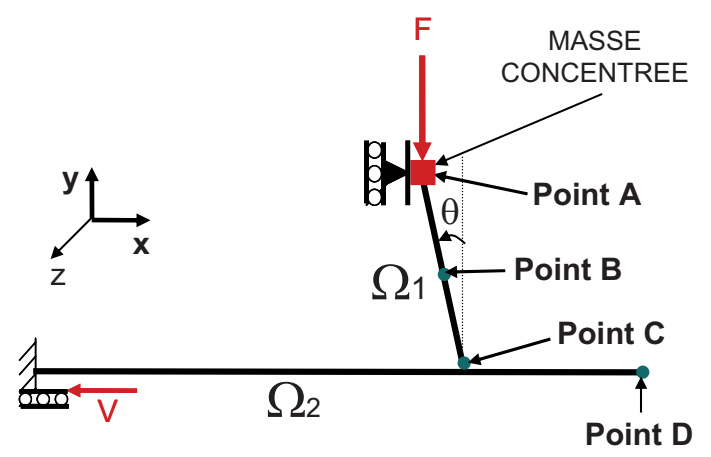

Fig. 1. Représentation du système modèle étudié. Initialement, le point $\mathrm{C}$ se trouve à $80 \mathrm{~mm}$ de l'encastrement de $\Omega_{2}$ pour les simulations numériques et les essais présentés.

d'analyses non-linéaires [19] qui permettent de traiter ces problèmes de vibrations induites par frottement, mais dans ces analyses, le plus souvent, de fortes hypothèses sont faites au niveau du contact (contact permanent, état glissant dans la même direction...) qui ne permettent pas d'étudier localement le comportement du système dans la zone de contact.

Le plus souvent, ces analyses numériques sont faites séparément. Quelques travaux couplent les deux analyses : l'analyse temporelle fournit les conditions de contact en fonctionnement dans un premier temps qui sont utilisées comme données d'entrée de l'analyse aux valeurs propres du système. Cette dernière donne alors l'allure du mode mis en jeu lors de l'instabilité [20-23]. Vola [24] effectue les deux types d'analyse en parallèle sur un système de balai d'essuie-glace/vitre. L'état statique glissant est utilisé dans l'analyse aux valeurs propres pour la recherche des modes instables et dans l'analyse temporelle dynamique comme condition initiale. De récentes études numériques de systèmes disque-plaquette mettant en place les deux analyses ont été réalisées par Abu Bakar [25] et Massi [26] montrent que l'analyse aux valeurs propres prédit plus de fréquences instables que l'analyse temporelle. Massi [26] montre également des différences de prédiction du domaine de stabilité des deux analyses mises en place. Dans la littérature, à notre connaissance, aucune étude portant sur les vibrations induites par frottement ne présente des résultats issus des deux analyses avec une validation expérimentale. C'est l'objectif de cet article qui, compte tenu de la complexité du phénomène d'instabilité de contact, porte sur l'étude des instabilités de contact d'un système modèle (Fig. 1). Dans un premier temps, les modélisations des deux analyses sont exposées. Puis les résultats issus des deux analyses sont exposés et synthétisés, afin de mettre en avant l'apport de chacune des deux analyses dans l'étude des instabilités de contact. Puis, dans un deuxième temps, l'étude expérimentale du système est effectuée et permet de valider les résultats numériques obtenus.

\section{Modélisation et méthodes numériques utilisées}

\subsection{Système étudié et équations de mouvement sans prise en compte du contact}

Le système étudié est constitué de deux poutres en acier $\Omega_{1}$ et $\Omega_{2}$ en contact ponctuel (Fig. 1). $\Omega_{2}$ est encastrée libre. Le point $\mathrm{A}$ de $\Omega_{1}$ est bloqué en translation suivant $x$ et en rotation suivant $z$. Une masse concentrée de $3 \mathrm{~kg}$ est prise en compte dans les calculs au point $\mathrm{A}$ et correspond au support physique de $\Omega_{1}$ permettant d'assurer la condition limite (voir Fig. 12). Une vitesse $V$ suivant $x$ est imposée à $\Omega_{2}$ au niveau de l'encastrement et la force $F$ est appliquée au point A suivant $y$.

La discrétisation spatiale est effectuée à partir de la méthode des éléments-finis. Les éléments considérés sont des éléments de poutre de Bernoulli (cisaillement négligé) en flexion et traction-compression (3 degrés de liberté par nœud). On se place dans les hypothèses linéaires de petites rotations et de petites déformations. Les équations de mouvement, via le principe des travaux virtuels, sont transformées en un système matriciel linéaire :

$$
\mathrm{M} \ddot{u}+\mathrm{C} \dot{u}+\mathrm{K} u=f
$$

\subsection{Analyse aux valeurs propres}

Le principe de cette analyse est de calculer les valeurs et vecteurs propres du système en intégrant les forces de contact dans la matrice $\mathbf{K}$. On écrit les équations de mouvement autour de l'équilibre glissant du système. La gestion du contact est réalisée par l'intermédiaire d'un ressort de raideur $K_{\mathrm{c}}$ introduit entre les deux nouds en contact (Fig. 2). Les deux nœuds en contact sont supposés cinématiquement glissants. Les forces de contact en chaque nœud en contact peuvent donc être exprimées ainsi :

$$
\left\{\begin{array}{l}
\lambda_{\Omega_{1}}^{\vec{n}}\left(\boldsymbol{u}^{\mathrm{p}}\right)=-\mathrm{K}_{\mathrm{c}} u_{\mathrm{rel}}^{\vec{n}} \\
\lambda_{\Omega_{1}}^{\vec{t}}\left(\boldsymbol{u}^{\mathrm{p}}\right)=-\mu\left|\lambda_{\Omega_{1}}^{\vec{n}}\right| \operatorname{sgn}\left(\nu_{\mathrm{rel}}^{\vec{t}}\right)
\end{array}\right.
$$

où $\lambda_{\Omega_{1}}^{\vec{n}}$ et $\lambda_{\Omega_{1}}^{\vec{t}}$ sont les valeurs des forces normale et tangentielle de contact respectivement sur la poutre $\Omega_{1}$.

$\boldsymbol{u}^{\mathrm{p}}$ représente le vecteur déplacement perturbé autour de la position d'équilibre glissant du système, $u_{\text {rel }}^{\vec{n}}$ est le déplacement relatif suivant $\vec{n}$ des deux poutres au niveau du contact, $v_{\text {rel }}^{\vec{t}}$ la vitesse tangentielle relative entre les deux nœuds en contact.

Les vecteurs nodaux des forces normales et tangentielles $\left(\lambda^{\vec{n}}\left(\boldsymbol{u}^{\mathrm{p}}\right)\right.$ et $\left.\lambda^{\vec{t}}\left(\boldsymbol{u}^{\mathrm{p}}\right)\right)$ peuvent donc être exprimés en fonction du coefficient de frottement de Coulomb, de la raideur du ressort et des déplacements nodaux. On obtient ainsi l'équation suivante :

$$
\begin{array}{r}
\mathbf{M} \ddot{\boldsymbol{u}}^{\mathrm{p}}+\mathbf{C} \dot{\boldsymbol{u}}^{\mathrm{p}}+\mathbf{K} \boldsymbol{u}^{\mathrm{p}}=\lambda^{\vec{n}}\left(\boldsymbol{u}^{\mathrm{p}}\right)+\lambda^{\vec{t}}\left(\boldsymbol{u}^{\mathrm{p}}\right) \Leftrightarrow \\
\mathbf{M} \ddot{\boldsymbol{u}}^{\mathrm{p}}+\mathbf{C} \dot{\boldsymbol{u}}^{\mathrm{p}}+\mathbf{K}^{\mathrm{c}} \boldsymbol{u}^{\mathrm{p}}=\mathbf{0}
\end{array}
$$




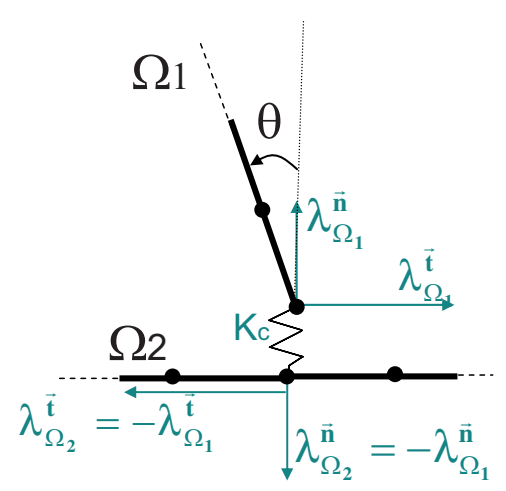

Fig. 2. Gestion du contact dans l'analyse aux valeurs propres. $\lambda_{\Omega_{1}}^{\vec{n}}$ et $\lambda_{\Omega_{1}}^{\vec{t}}$ représente les forces normale et tangentielle appliquées sur la poutre $\Omega_{1}$.

Les valeurs propres $\varphi_{i}$ complexes et conjuguées deux à deux du système (3) sont

$$
\varphi_{i}=\varepsilon_{i} \omega_{i}+j \omega_{i}
$$

Lorsque $\varepsilon_{i}$ est négatif, $y$ croît exponentiellement et l'équilibre glissant du système est instable par flottement.

\subsection{Analyse temporelle}

Les valeurs de déplacements, vitesses et accélérations aux différents nœuds, ainsi que les forces et surfaces de contact au cours du temps sont obtenues. La gestion du contact avec frottement entre les deux corps déformables est réalisée par un algorithme basé sur la méthode des multiplicateurs de Lagrange à incrément avant [27]. L'algorithme de contact est basé sur l'interaction entre les surfaces maîtres (sur $\Omega_{2}$ ) et le nœud esclave (point C appartenant à $\Omega_{1}$ ). Les segments maîtres élémentaires sont décrits par deux nouds et approximés par des splines bicubiques de continuité $\mathrm{C}^{1}$. La méthode des multiplicateurs de Lagrange à incrément avant est construite à partir des équations de mouvement (via le principe des travaux virtuels) au temps $\left(t_{n}=n \Delta t\right)$ augmentées par les conditions en déplacements imposées au nœud esclave à l'instant $t_{n+1}$ :

$$
\left\{\begin{array}{l}
\boldsymbol{M} \ddot{\boldsymbol{u}}_{n}+\boldsymbol{C} \dot{\boldsymbol{u}}_{n}+\boldsymbol{K} \boldsymbol{u}_{n}=\boldsymbol{G}_{n+1}^{\boldsymbol{T}} \boldsymbol{\lambda}_{n}=\boldsymbol{f}_{n} \\
\mathbf{G}_{n+1}\left(\boldsymbol{x}_{n}+\boldsymbol{u}_{n+1}+\boldsymbol{u}_{n}\right)=\mathbf{G}_{n+1} \boldsymbol{x}_{x+1} \leq 0
\end{array}\right.
$$

Les équations de mouvement (6) sont ensuite discrétisées en temps. On choisit un schéma de Newmark explicite $\beta_{2}$ (ce qui impose que la condition de Courant soit satisfaite). Les vecteurs $\dot{\boldsymbol{u}}_{n}$ et $\ddot{\boldsymbol{u}}_{n}$ sont exprimés à chaque pas de temps $\Delta t$ à partir du schéma temporel de la méthode $\beta_{2}$ $\left(\beta_{2} \in[0,5 ; 1[):\right.$

$$
\left\{\begin{array}{l}
\ddot{\boldsymbol{u}}=\frac{2}{\Delta t^{2}}\left(\boldsymbol{u}_{n+1}-\boldsymbol{u}_{n}+\Delta t \dot{\boldsymbol{u}}_{n}\right) \\
\dot{\boldsymbol{u}}+\frac{1}{1+2 \beta_{2}}\left\{\dot{\boldsymbol{u}}_{n-1}+\Delta t\left(1-\beta_{2}\right) \ddot{\boldsymbol{u}}_{n-1}+\frac{2 \beta_{2}}{\Delta t}\left(\boldsymbol{u}_{n+1}-\boldsymbol{u}_{n}\right)\right\}
\end{array}\right.
$$

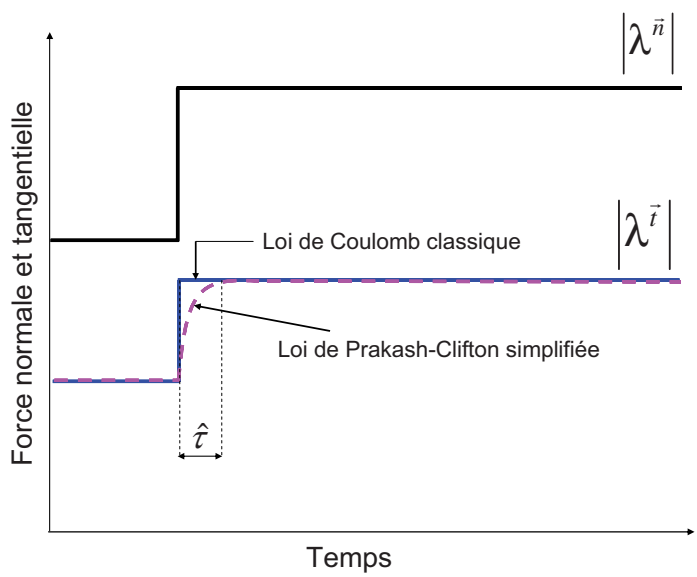

Fig. 3. Représentation de la loi de Coulomb classique et de la loi de Coulomb régularisée choisie (loi de Prakash-Clifton simplifiée). La force tangentielle donnée par la loi de Coulomb classique répond instantanément à une variation brutale de la force normale, contrairement à celle donnée par la loi de Prakash-Clifton simplifiée.

La loi de frottement utilisée au contact est la loi simplifiée du type Prakash-Clifton [28, 29] (Fig. 3). Les conditions de contact sont données par :

$\lambda_{\mathrm{n}}^{\vec{n}} \leq 0\left(\right.$ contact si $\lambda_{\mathrm{n}}^{\vec{n}}<0$ et décollement si $\left.\lambda_{\mathrm{n}}^{\vec{n}}=0\right)$.

Dans le cas où $\lambda_{\mathrm{n}}^{\vec{n}}<0:\left|\lambda_{\mathrm{n}}^{\vec{t}}\right| \leq \mu\left|\lambda_{\mathrm{n}}^{\vec{n}}\right|$

- Si on a $\left|\lambda_{\mathrm{n}}^{\vec{t}}\right|_{\mathrm{c}}<\mu\left|\lambda_{\mathrm{n}}^{\vec{n}}\right|$, alors $\nu_{\text {rel }}^{\vec{t}}=0$ (adhérence) et à $t_{n},\left|\lambda_{\mathrm{n}}^{\vec{t}}\right|=\left|\lambda_{\mathrm{n}}^{\vec{t}}\right|_{\text {calc }}$.

- Si on a $\left|\lambda_{\mathrm{n}}^{\vec{t}}\right|_{\mathrm{c}} \geq \mu\left|\lambda_{\mathrm{n}}^{\vec{n}}\right|$, alors $\lambda_{\mathrm{n}}^{\vec{t}} \cdot \nu_{\text {rel }}^{\vec{t}} \leq 0$ (glissement) (8)

$$
\dot{\lambda}^{\vec{t}}=-\frac{\hat{V}}{\hat{L}}\left(\left|\lambda^{\vec{t}}\right|-\mu\left|\lambda^{\vec{n}}\right|\right) \quad \text { et } \quad \grave{a} \quad t_{n}, \quad\left|\lambda_{\mathrm{n}}^{\vec{t}}\right|=
$$
$\frac{1}{1+\frac{\hat{V}}{L} \Delta t}\left(\left|\lambda_{\mathrm{n}-1}^{\vec{t}}\right|+\frac{\hat{V}}{\hat{L}} \Delta t \mu\left|\lambda_{\mathrm{n}}^{\vec{n}}\right|\right)$ où $v_{\mathrm{rel}}^{\vec{t}} \quad$ est la vitesse relative tangentielle des nœuds esclaves liés à la surface maître. $\dot{\lambda}^{\vec{t}}$ est la dérivée de la force tangentielle de contact en fonction du temps.

La force tangentielle atteint la force tangentielle de Coulomb $\left|\lambda_{\mathrm{n}}^{\vec{t}}\right|=\mu\left|\lambda_{\mathrm{n}}^{\vec{n}}\right|$ après un glissement sur une longueur $\hat{L}$, ou après un temps $\hat{\tau}$ qui vaut $\frac{\hat{V}}{\hat{L}} \cdot\left|\lambda_{\mathrm{n}}^{\vec{t}}\right|_{\mathrm{c}}$ correspond à la valeur de la force tangentielle calculée avant l'application des conditions de contact.

\section{2 Étude numérique et confrontation des deux types d'analyses}

Les caractéristiques du système étudié figurent dans le tableau 1. Le modèle numérique contient 120 élémentsfinis (soit 122 nœuds et 366 degrés de liberté). Pour modéliser correctement le système dans le domaine fréquentiel d'intérêt [0-10 kHz], une longueur d'élément 
Tableau 1. Données du système étudié.

\begin{tabular}{lll}
\hline & $\Omega_{1}$ & $\Omega_{2}$ \\
\hline Longueur & $0,05 \mathrm{~m}$ & $0,15 \mathrm{~m}$ \\
Épaisseur & $0,01 \mathrm{~m}$ & $0,015 \mathrm{~m}$ \\
Largeur & $0,0015 \mathrm{~m}$ & $0,003 \mathrm{~m}$ \\
Masse volumique $(\rho)$ & $7900 \mathrm{~kg} \cdot \mathrm{m}^{-3}$ & $7900 \mathrm{~kg} \cdot \mathrm{m}^{-3}$ \\
Module d'Young $(E)$ & $185 \mathrm{GPa}$ & $185 \mathrm{GPa}$ \\
Paramètre $\alpha_{1}$ & $50 \mathrm{~s}^{-1}$ & $50 \mathrm{~s}^{-1}$ \\
Paramètre $\alpha_{2}$ & $1 \times 10^{-8} \mathrm{~s}$ & $1 \times 10^{-8} \mathrm{~s}$ \\
Nombre d'éléments & 30 & 90 \\
\hline
\end{tabular}

de 1,7 mm a été choisie. Cette longueur est bien inférieure à dix fois la longueur d'onde $\left(c / f_{\max } \approx 50 \mathrm{~mm}\right)$. Les coefficients $\alpha_{1}$ et $\alpha_{2}$ ont été obtenus lors de l'étude expérimentale de la poutre $\Omega_{2}$ seule. Pour les premiers modes de la structure assemblée, il a été vérifié que les taux d'amortissement appliqués numériquement et mesurés expérimentalement sont proches, surtout pour les fréquences proches de la fréquence de crissement. L'angle $\theta$ est fixé à $5^{\circ}$. Dans les simulations présentées, le contact entre les deux poutres s'effectue au point $C$ (Fig. 1). Les surfaces de contact sont considérées comme étant parfaitement lisses. Les effets thermiques et physicochimiques sont négligés.

\subsection{Analyse aux valeurs propres}

Une étude de sensibilité à la valeur de la raideur de contact $K_{\mathrm{c}}$ a été effectuée. Pour des valeurs du même ordre de grandeur que la raideur des éléments du système, les résultats (domaine d'instabilité, valeurs propres) sont sensibles à ce paramètre. Cependant, pour des valeurs grandes devant la raideur élémentaire du système, les résultats sont indifférents aux variations de $K_{\mathrm{c}}$, ce qui indique que, pour ces valeurs, le contact peut être considéré comme infiniment rigide. Dans la modélisation temporelle, l'utilisation de la méthode des « multiplicateurs de Lagrange à incrément avant $»$ implique que le contact est considéré comme infiniment rigide. Ainsi pour avoir des résultats comparables, la raideur de contact $K_{\mathrm{c}}$ est fixée à $10^{5}$ fois la raideur élémentaire maximale du système $\left(K_{\mathrm{c}}=1,35 \times 10^{14}\right.$ N.m $\left.{ }^{-1}\right)$.

L'instabilité se manifeste par une ou plusieurs coalescences de deux modes en fréquence (partie imaginaire de la valeur propre). Parallèlement, une des deux valeurs propres possède un taux d'amortissement $\varepsilon_{i}$ négatif. La figure $4 \mathrm{a}$ présente l'analyse aux valeurs propres du système en fonction du coefficient de frottement. Dans le domaine fréquentiel considéré et pour des coefficients de frottement compris entre 0 et 0,5 , on observe trois coalescences de modes $(400 \mathrm{~Hz}, 2800 \mathrm{~Hz}$ et $7700 \mathrm{~Hz})$ à des coefficients de frottement différents $\left(\mu_{\mathrm{c}}^{400 \mathrm{~Hz}}=0,16\right.$, $\mu_{\mathrm{c}}^{2800 \mathrm{~Hz}}=0,12$ et $\left.\mu_{\mathrm{c}}^{7700 \mathrm{~Hz}}=0,23\right)$.

La forme des modes (Figs. 4b-d) et le glissement relatif au niveau du contact sont des paramètres importants de la coalescence de modes liée à l'instabilité [13,26]. Par exemple, le mode (9) $(8070 \mathrm{~Hz}$ à $\mu=0)$ qui est un mode
Tableau 2. Paramètres des simulations temporelles présentées.

\begin{tabular}{ll}
\hline Force appliquée $(F)$ & $9 \mathrm{~N}$ \\
Vitesse appliquée $(V)$ & $0,002 \mathrm{~m} \cdot \mathrm{s}^{-1}$ \\
Pas de temps $(\Delta T)$ & $2 \times 10^{-8} \mathrm{~s}$ \\
Amortissement numérique $\left(\beta_{2}\right)$ & 0,8 \\
\hline
\end{tabular}

de traction pure de $\Omega_{2}$ et qui présente un nœud de vibration au point de contact, ne subit aucun couplage avec le mode (8) (7180 Hz à $\mu=0)$ ou le mode (10) $(8350 \mathrm{~Hz}$ à $\mu=0)$.

\subsection{Analyse temporelle}

Les paramètres des simulations présentées sont donnés dans le tableau 2. Les paramètres de maillage et de pas de temps ont été définis afin d'obtenir la convergence des résultats en temps et en espace. Les paramètres de régularisation sont fixés $\left(\hat{V}=V\right.$ et $\left.\hat{L}=2 \times 10^{-10} \mathrm{~m}\right)$. À l'instant initial, les poutres sont immobiles et ne sont pas en contact. $\Omega_{1}$ est alors mise en mouvement suivant $y$ par la force $F$ appliquée et les deux poutres entrent en contact (phase I $: t \in[0 \mathrm{~s} ; 0,4 \mathrm{~s}]$ ). Lorsque les deux poutres en contact sont en équilibre statique, on applique une vitesse $V$ suivant $x$ à $\Omega_{2}$ (phase II $: t \in[0,4 \mathrm{~s} ; 1 \mathrm{~s}]$ ).

\subsection{1 Équilibre glissant}

Les figures 5 et 6 présentent la vitesse suivant $x$ du nœud esclave (point $\mathrm{C}$ ) et la force de contact suivant $y$ en fonction du temps pour un coefficient de frottement de 0,1 et $V=-2 \mathrm{~mm} \cdot \mathrm{s}^{-1}$. Lors de la mise en mouvement de $\Omega_{2}$, un régime vibratoire transitoire de très faible amplitude se met en place et laisse rapidement place à un régime stable glissant.

La vitesse du nœud esclave tend vers zéro et la force de contact suivant $y$ tend vers $9 \mathrm{~N}$, valeur absolue de la force $F$ imposée. Les valeurs locales de vitesse et de force tendent vers les valeurs globalement imposées.

\subsubsection{Apparitions d'instabilités de contact}

Lorsqu'on augmente le coefficient de frottement, le système passe d'un régime glissant stable à un régime d'instabilité de contact. Pour les paramètres définis dans le tableau 2, le coefficient de frottement critique au-dessus duquel l'instabilité est générée vaut 0,12. Les figures 7 et 8 présentent la vitesse suivant $x$ des nouds B et $\mathrm{C}$ et les forces de contact suivant $x$ et $y$ en fonction du temps pour un coefficient de frottement de 0,15 . Le phénomène d'instabilité de contact se traduit par un phénomène de vibrations auto-entretenues du système. L'amplitude des vibrations est plus importante que dans le cas où le système est stable. La figure 9 présente la transformée de Fourier des forces de contact suivant $x$ et $y$. Le spectre présente 


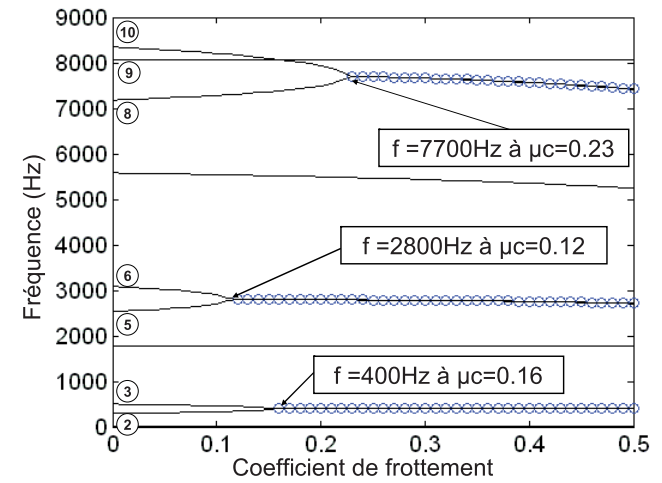

(a)

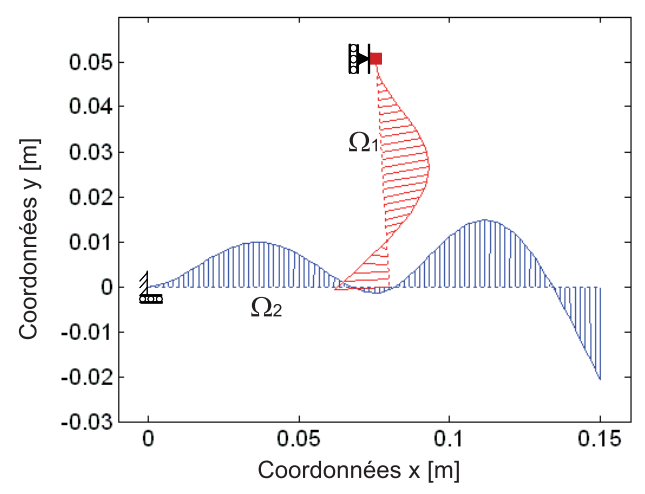

(c) Mode (5) $(\mu=0,11$ et $f=2800 \mathrm{~Hz})$

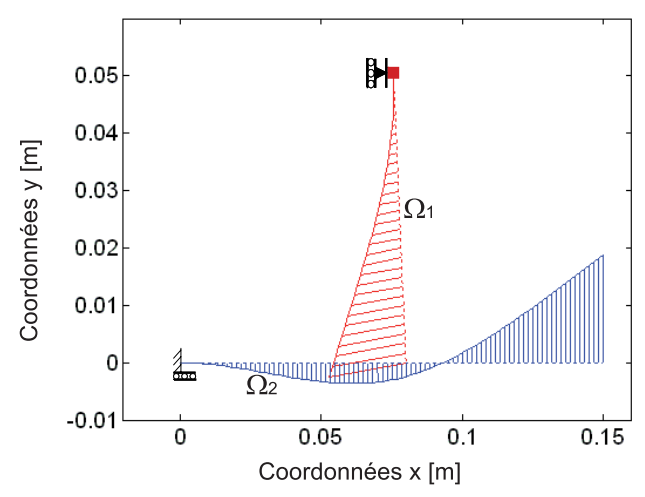

(b) Mode (2) ( $\mu=0,15$ et $f=380 \mathrm{~Hz})$

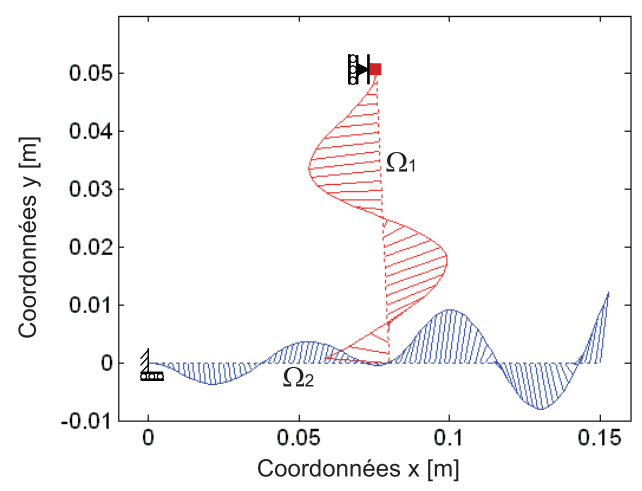

(d) Mode 8 ( $\mu=0,22$ et $f=7620 \mathrm{~Hz})$

Fig. 4. Analyse aux valeurs propres numérique du système : (a) évolution des 10 premières fréquences du système couplé par frottement en fonction du coefficient de frottement $\left(\theta=5^{\circ}\right)(\bigcirc$ fréquences d'instabilité potentielle), (b) à (d) allures des modes 2,5 et 8 avec un coefficient de frottement $\mu$ de 0,01 en dessous du coefficient de frottement critique $\mu_{\mathrm{c}}\left(\mu=\mu_{\mathrm{c}}-0,01\right)$.

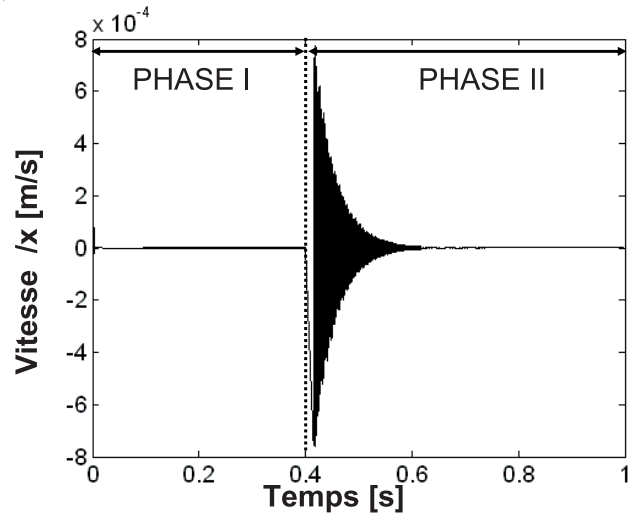

Fig. 5. Vitesse suivant $x$ du noud esclave (point C). (Simulation numérique temporelle avec $\mu=0,1$ et $V=-2 \mathrm{~mm} . \mathrm{s}^{-1}$.)

une périodicité en fréquence dont la fréquence principale se trouve à $2700 \mathrm{~Hz}$.

L'apparition de vibrations auto-entretenues se manifeste par l'apparition d'ondes au niveau du contact qui sont de type adhérence-glissement, adhérenceglissement-décollement ou glissement-décollement [16, $30-32$. Il y a donc localement des variations du statut

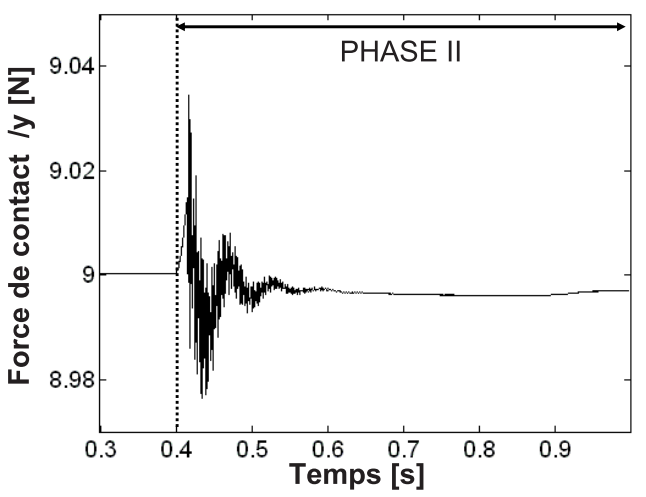

Fig. 6. Force de contact suivant $y$ pendant la deuxième phase de simulation (simulation numérique temporelle avec $\mu=0,15$ et $\left.V=-2 \mathrm{~mm} \cdot \mathrm{s}^{-1}\right)$.

cinématique (adhérent, glissant, décollé) du nœud esclave par rapport à la surface de contact, ainsi que des variations de la force de contact (normale et tangentielle), des vitesses... Dans la simulation présentée, ce sont des ondes de type adhérence-glissement-décollement à la fréquence de $2700 \mathrm{~Hz}$ qui sont présentes au niveau du 


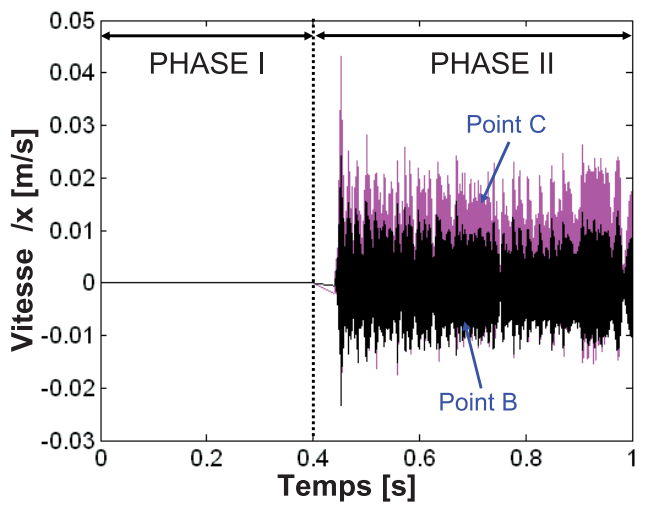

Fig. 7. Vitesse suivant $x$ des points $\mathrm{B}$ et $\mathrm{C}$ (simulation numérique temporelle avec $\mu=0,15, F=9 \mathrm{~N}$ et $V=$ $\left.-2 \mathrm{~mm} \cdot \mathrm{s}^{-1}\right)$.

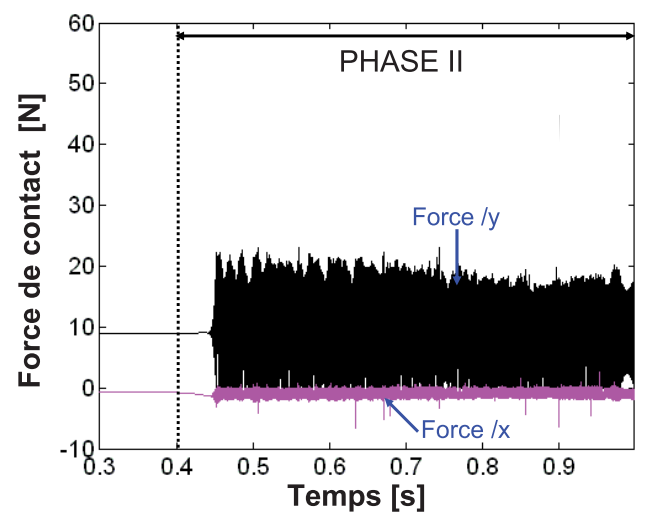

Fig. 8. Forces de contact selon $x$ et $y$ (simulation numérique temporelle avec $\mu=0,15, F=9 \mathrm{~N}$ et $V=-2 \mathrm{~mm} \cdot \mathrm{s}^{-1}$ ).

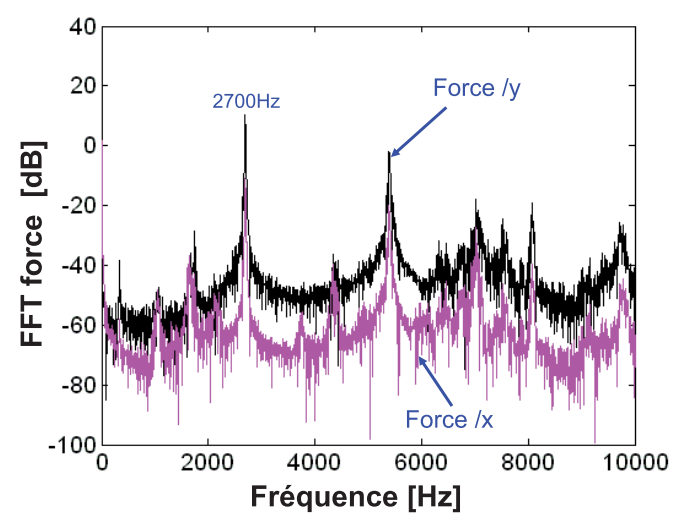

Fig. 9. FFT des forces de contact selon $x$ et $y$ (simulation numérique temporelle avec $\mu=0,15 F=9 \mathrm{~N}$ et $V=$ $\left.-2 \mathrm{~mm} . \mathrm{s}^{-1}\right)$.

contact. Localement, les conditions de contact sont plus sévères que celles imposées globalement. En effet, la force de contact peut atteindre $23 \mathrm{~N}$ au lieu des $9 \mathrm{~N}$ imposé globalement. De même, alors que la valeur de la vitesse relative macroscopique est de $2 \mathrm{~mm} . \mathrm{s}^{-1}$, la vitesse relative locale peut atteindre plus de $40 \mathrm{~mm} . \mathrm{s}^{-1}$.

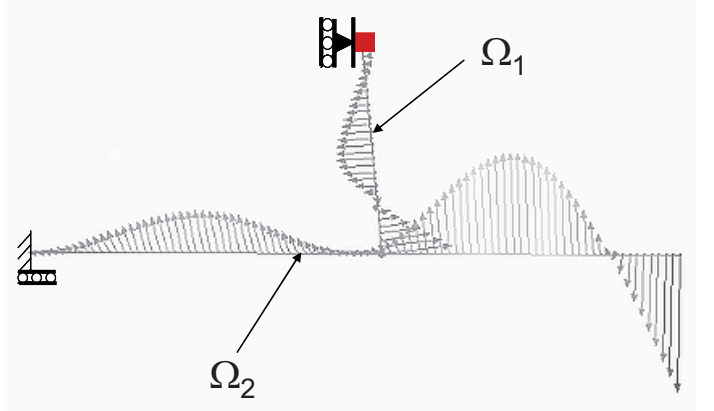

Fig. 10. Représentation des vecteurs vitesses en chaque nœud du système à $t=1 \mathrm{~s}$ (simulation numérique temporelle avec $\mu=0,15, F=9 \mathrm{~N}$ et $\left.V=-2 \mathrm{~mm} \cdot \mathrm{s}^{-1}\right)$. Résultats similaires à la figure $4 \mathrm{c}$ obtenue par l'analyse aux valeurs propres.

La figure 10 présente les vecteurs vitesses de chaque nœud du système pendant l'instabilité à $t=1 \mathrm{~s}$. Cette représentation montre que le système vibre selon un mode typique de la structure assemblée (mode obtenu lors de l'analyse aux valeurs propres Fig. $4 \mathrm{c}$ ), car $\Omega_{2}$ présente des vibrations dont l'allure ne correspond pas à celle d'un mode de poutre encastrée-libre. Les vibrations liées au crissement (type particulier d'instabilités de contact qui se caractérise par des vibrations instables continues et harmoniques de hautes fréquences [3]) sont dues à des vibrations couplées des deux structures en contact.

\subsection{Synthèse des résultats des deux analyses}

Dans le mouvement décrit par le système, la position du point de contact évolue au cours du temps. L'analyse temporelle simule le comportement du système en temps et prend ainsi en compte cette évolution. L'analyse aux valeurs propres ne tient pas compte de cette évolution du point de contact. Cependant, le déplacement du point de contact étant de l'ordre de 1,1 mm, il n'y a pas d'influence sur les résultats de l'analyse aux valeurs propres.

D'après l'analyse aux valeurs propres, le système est instable à partir d'un coefficient de frottement de 0,12 (Fig. 4a). Une valeur identique est obtenue par l'analyse temporelle.

Pour un coefficient de frottement $\mu$ de 0,15 , les deux analyses donnent les mêmes résultats : une seule fréquence instable à $2700 \mathrm{~Hz}$ pour l'analyse temporelle et $2800 \mathrm{~Hz}$ pour l'analyse aux valeurs propres. La représentation temporelle des vibrations du système (Fig. 10) pendant l'instabilité correspond au mode instable obtenu par l'analyse aux valeurs propres (Fig. 4c).

Pour des coefficients de frottement plus élevés, l'analyse aux valeurs propres donne plusieurs fréquences potentiellement instables. Les simulations temporelles indiquent que ces fréquences ne sont pas toutes instables. Pour un coefficient de frottement de 0,25, d'après les résultats de l'analyse aux valeurs propres, trois fréquences sont potentiellement instables : $400 \mathrm{~Hz}$, $2800 \mathrm{~Hz}$ et $7700 \mathrm{~Hz}$ (Fig. 4a). Les résultats des simulations temporelles indiquent que seul le mode à $2700 \mathrm{~Hz}$ 


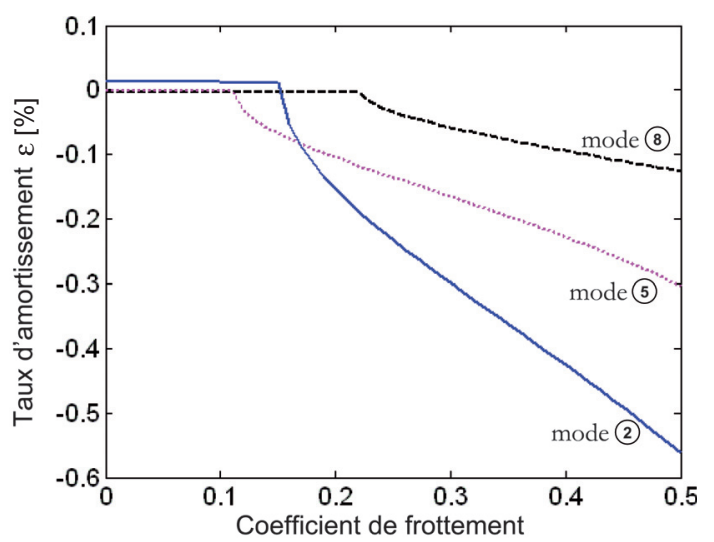

Fig. 11. Taux d'amortissement $\varepsilon$ pour les modes (2) (400 Hz), (5) $(2700 \mathrm{~Hz})$ et $8(7700 \mathrm{~Hz})$ en fonction du coefficient de frottement.

est instable pour les conditions de fonctionnement (vitesse $V$ de -2 mm.s ${ }^{-1}$, force $F$ de $\left.9 \mathrm{~N}\right)$. La figure 11 présente l'évolution des taux d'amortissement des modes (2), (5) et (8) (donnée par l'analyse aux valeurs propres) en fonction du coefficient de frottement. Dans certains travaux ([33] par exemple) basés sur une analyse aux valeurs propres, cette valeur du taux d'amortissement des modes potentiellement instables est considérée comme un bon indice pour déterminer le mode qui est excité en conditions réelles de fonctionnement. D'après cet indice (Fig. 11), à partir d'un coefficient de frottement de 0,17 , c'est le mode (2) qui devrait être prépondérant car il possède le taux d'amortissement le plus élevé en valeur absolue. Pourtant ce n'est pas le mode d'instabilité que prédit l'analyse temporelle. Ainsi, pour notre système, la valeur du taux d'amortissement des modes potentiellement instables n'est pas un bon indice pour déterminer le mode qui est excité en conditions réelles de fonctionnement. Ce taux d'amortissement permet uniquement de caractériser le début du mouvement dynamique.

Les deux analyses donnent donc des résultats très proches pour le domaine de stabilité. Cependant, l'analyse aux valeurs propres prédit, dans certains cas, plus de fréquences potentiellement instables que l'analyse temporelle. Une validation expérimentale est ensuite menée afin d'appuyer les résultats numériques obtenues par les deux analyses.

\section{Validation expérimentale}

\subsection{Banc d'essai}

Le banc utilisé est représenté sur la figure 12. La poutre $\Omega_{1}$ (1) est bloquée en translation selon $x$ et en rotation selon $z$ mais libre selon $y$. L'application physique de ces conditions limites impose la présence d'un support (3) (dont la masse est de $3 \mathrm{~kg}$ ) qui modifie la dynamique du système. (Ce support est modélisé par une masse concentrée dans les simulations numériques.) Le support de l'ensemble $\Omega_{1}$-support peut pivoter autour

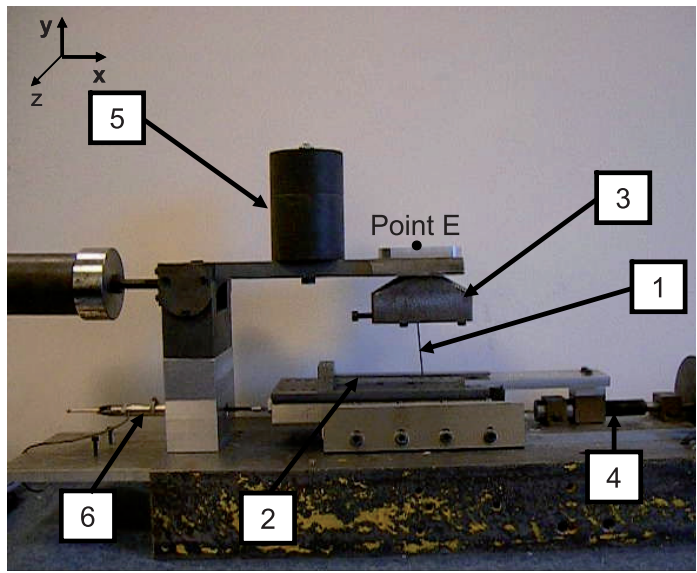

Fig. 12. Banc d'essai. (1) Poutre $\Omega_{1},(2)$ poutre $\Omega_{2}$, (3) support de la poutre $\Omega_{1}$, (4) système vis-écrou, (5) poids, (6) capteur de déplacement.

d'un axe solidaire du bâti. La force verticale $F$ de 9 N est appliquée par l'intermédiaire d'un poids (4). La poutre $\Omega_{2}$ (2) est encastrée-libre et repose sur un support coulissant suivant $x$ par rapport au bâti. Un système vis-écrou (4) permet de translater ce support.

Lors d'un essai en régime stable glissant $(V>0)$, le coefficient de frottement a été estimé entre 0,2 et 0,25 . L'étude expérimentale a été réalisée en deux temps. Dans un premier temps, une étude modale du système complet à l'arrêt a été réalisée afin de valider la modélisation dynamique du système sans mouvement relatif entre les deux poutres. Dans un second temps, l'étude des vibrations du système lorsque la vitesse $V(<0)$ est appliquée à $\Omega_{2}$ a été réalisée.

Afin de réaliser l'étude modale du système à l'arrêt, un pot excitateur muni d'un capteur de force piézoélectrique a été disposé sur le support de $\Omega_{1}$ au niveau du point $\mathrm{E}$ (Fig. 12). Il est maintenu inactif pendant l'étude des vibrations du système lorsque la vitesse $V$ est appliquée à $\Omega_{2}$ afin de ne pas modifier la dynamique du système. Ce capteur piézoélectrique enregistre la force au niveau du point $\mathrm{E}$ en fonction du temps. Un vibromètre laser à fibre optique permet de mesurer la vitesse de vibration suivant la direction de visée d'un point du système. Un capteur de déplacement (5) repère la position du plateau support de $\Omega_{2}$ et permet ainsi de connaître la vitesse de translation du plateau.

\section{2 Étude modale du système à l'arrêt}

Avant d'étudier la dynamique du système en frottement, il est nécessaire de vérifier que la dynamique du système au repos $(V=0)$ est bien modélisée. Pour cela, une étude modale du système à l'arrêt a été effectuée afin de comparer les modes et fréquences propres numériques et expérimentales du système. Expérimentalement, dans un premier temps, on soumet la structure à un bruit blanc afin d'obtenir les fréquences propres du système. La forme des modes expérimentaux est obtenue grâce à la mesure des niveaux et phases vibratoires en différents 


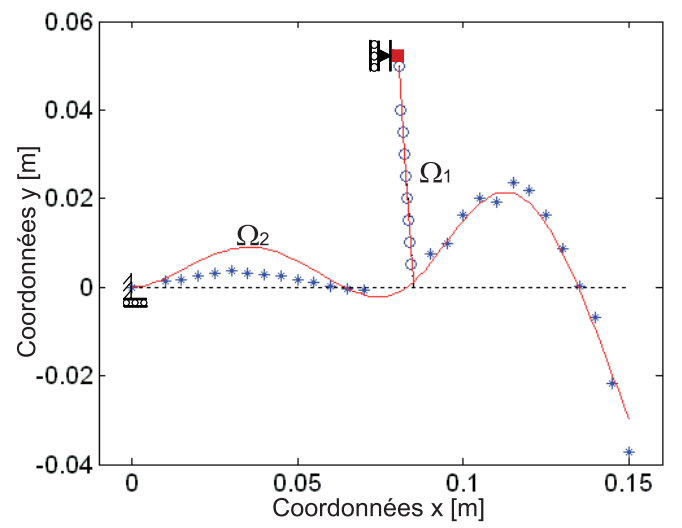

(a)

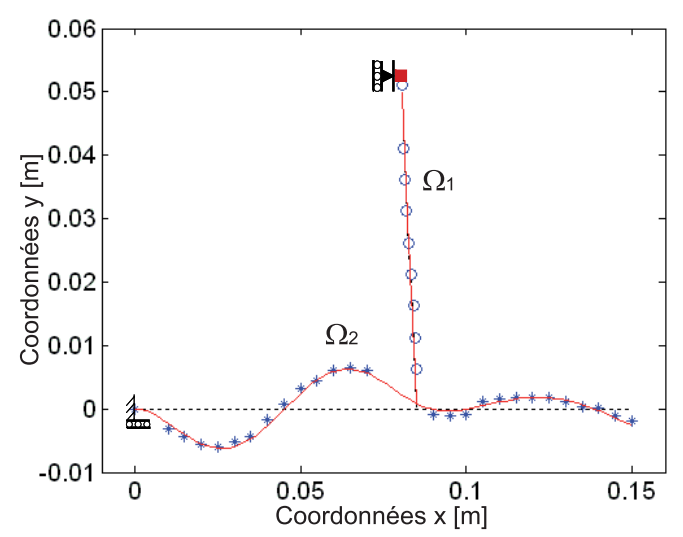

(b)

Fig. 13. Représentation numérique et expérimentale des modes du système couplé (a) $2930 \mathrm{~Hz}$ (b) $4875 \mathrm{~Hz}$. Représentation des poutres non déformées, $\bigcirc$ amplitude modale expérimentale pour les points de $\Omega_{1}$, ${ }^{*}$ amplitude modale expérimentale pour les points de $\Omega_{2}$, mode numérique.

points du système soumis à une excitation sinusoïdale aux fréquences propres expérimentales.

Ces modes sont ensuite comparés aux modes numériques obtenus par une recherche classique de modes propres des deux poutres collées au niveau contact. La figure 13 présente deux des modes du système à l'arrêt. Tous les modes obtenus expérimentalement ont été identifiés avec une bonne précision. Les fréquences expérimentales et numériques sont présentées dans le tableau 3. L'erreur sur les fréquences n'excède pas $4 \%$. La modélisation dynamique du système expérimental présenté figure 12 est donc validée.

\section{3 Étude des vibrations du système lorsque $\Omega_{2}$ est en translation}

Chaque essai se déroule en trois étapes : d'abord, la surface de $\Omega_{2}$ est nettoyée afin d'avoir des conditions de contact reproductibles. Puis, les poutres sont mises en contact. Enfin, lorsque le système est en équilibre
Tableau 3. Fréquences propres numériques et expérimentales et erreur relative.

\begin{tabular}{lll}
\hline $\begin{array}{l}\text { Fréquences } \\
\text { numériques }\end{array}$ & $\begin{array}{l}\text { Fréquences } \\
\text { expérimentales }\end{array}$ & $\begin{array}{l}\text { Erreur relative sur } \\
\text { la valeur de fréquence }\end{array}$ \\
\hline $16 \mathrm{~Hz}$ & $16 \mathrm{~Hz}$ & $0 \%$ \\
$362 \mathrm{~Hz}$ & $374 \mathrm{~Hz}$ & $3,2 \%$ \\
$1665 \mathrm{~Hz}$ & $1620 \mathrm{~Hz}$ & $2,8 \%$ \\
$2060 \mathrm{~Hz}$ & $2080 \mathrm{~Hz}$ & $0,9 \%$ \\
$2924 \mathrm{~Hz}$ & $2930 \mathrm{~Hz}$ & $0,1 \%$ \\
$5035 \mathrm{~Hz}$ & $4875 \mathrm{~Hz}$ & $3,3 \%$ \\
$6624 \mathrm{~Hz}$ & $6600 \mathrm{~Hz}$ & $0,4 \%$ \\
$8030 \mathrm{~Hz}$ & $7690 \mathrm{~Hz}$ & $4 \%$ \\
$8236 \mathrm{~Hz}$ & $8100 \mathrm{~Hz}$ & $2 \%$ \\
\hline
\end{tabular}

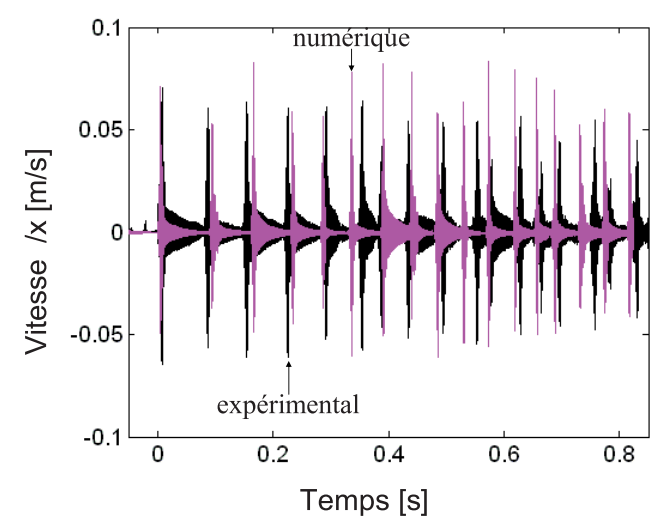

Fig. 14. Vitesse numérique et expérimentale suivant $x$ au point $\mathrm{B}$ en fonction du temps $\left(V=-2 \mathrm{~mm} \cdot \mathrm{s}^{-1}\right.$ et $\left.F=9 \mathrm{~N}\right)$.

statique, $\Omega_{2}$ est mise en mouvement. C'est lors de cette dernière phase que les mesures suivantes sont effectuées :

- la mesure de la force $F_{\mathrm{E}}(t)$ mesurée au point $\mathrm{E}$ par le capteur piézoélectrique lié au pot excitateur inactif,

- la mesure de la vitesse $v_{\mathrm{B}}(t)$ du point B (Fig. 1).

La figure 14 présente les vitesses suivant $x$ numérique et expérimentale $\mathrm{du}$ point $\mathrm{B}$ en fonction du temps pour une vitesse de translation de $\Omega_{2}$ de -2 mm.s ${ }^{-1}$. Numériquement et expérimentalement, le système présente des instabilités de contact et vibre de manière discontinue. Numériquement, les séquences d'instabilité apparaissent à intervalles de temps régulier. L'intervalle de temps (environ 0,04 s) entre deux phases d'instabilité correspond au temps pendant lequel $\Omega_{2}$ entraîne $\Omega_{1}$ par adhérence. C'est ensuite le glissement entre $\Omega_{2}$ et $\Omega_{1}$ qui met le système en vibrations. Ces intervalles de temps sont liés à la vitesse de $\Omega_{2}$. On peut observer que les intervalles de temps numériques et expérimentaux entre les séquences d'instabilité sont proches.

Les figures 15 et 16 présentent les spectres des vitesses numérique et expérimentale suivant $x$ du point $\mathrm{B}$ (Fig. 1) et les spectres de la force de contact numérique (direction $y$ ) et de la force $F_{\mathrm{E}}$ (direction $y$ ) expérimentale pour une vitesse de translation de $\Omega_{2}$ de $-2 \mathrm{~mm} . \mathrm{s}^{-1}$. Expérimentalement, la force de contact ne peut pas être mesurée. Cependant, les vibrations du système qui se propagent jusqu'au capteur, sont représentatives 


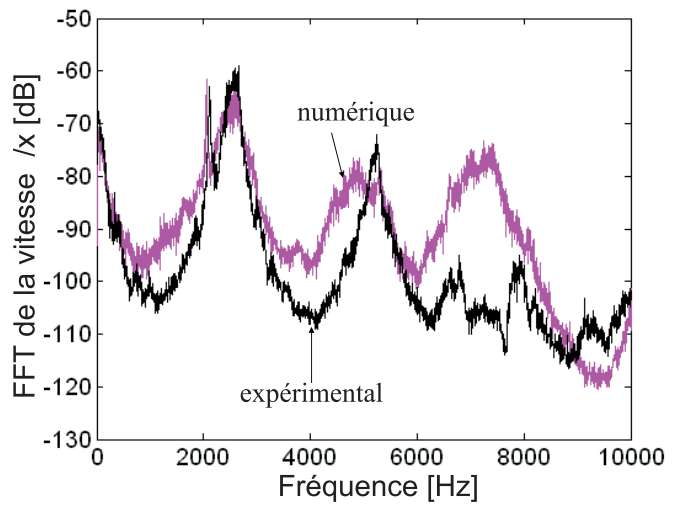

Fig. 15. FFT des vitesses numérique et expérimentale suivant $x$ du point $\mathrm{B}\left(V=-2 \mathrm{~mm} . \mathrm{s}^{-1}\right.$ et $\left.F=9 \mathrm{~N}\right)$.

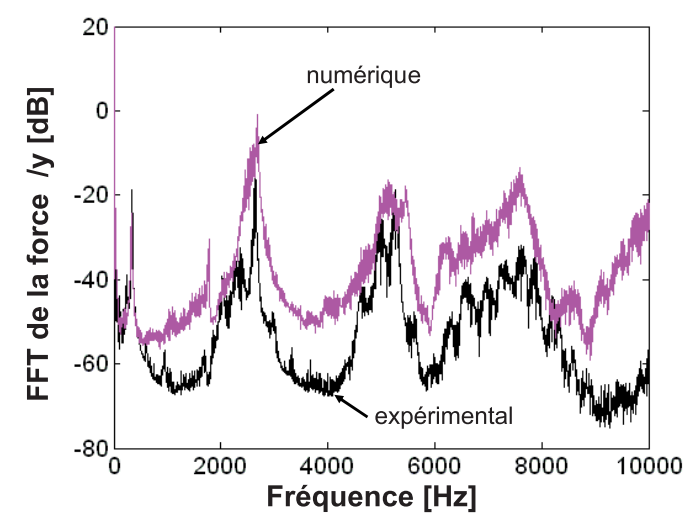

Fig. 16. FFT de la force de contact numérique suivant $y$ numérique et de $F_{\mathrm{E}}$ expérimentale $\left(V=-2 \mathrm{~mm} \cdot \mathrm{s}^{-1}\right.$ et $F=9 \mathrm{~N})$.

des vibrations de la force de contact du point de vue de leur contenu fréquentiel. D'après ces figures, expérimentalement, les vibrations du système présentent une périodicité en fréquence, avec comme fréquence principale $2700 \mathrm{~Hz}$ (Figs. 15, 16), ce qui est également prédit par les simulations numériques. De plus, l'amplitude numérique des vibrations en temps et en fréquence est très proche de celle obtenue expérimentalement. Ceci montre que la fréquence d'instabilité ainsi que son niveau de vibration sont bien prédits numériquement par l'analyse temporelle. L'analyse aux valeurs propres prédit deux fréquences d'instabilité à un coefficient de frottement $\mu$ de $0,2: 400 \mathrm{~Hz}$ et $2800 \mathrm{~Hz}$. Seule la dernière (environ $2800 \mathrm{~Hz}$ ) apparaît dans le spectre expérimental, ce qui valide en partie l'analyse aux valeurs propres puisque la fréquence de vibration expérimentale est prédite. Mais l'analyse aux valeurs propres n'est pas suffisante car elle surestime le nombre de fréquences d'instabilité.

\section{Conclusions}

Cet article présente l'étude des instabilités de contact sur un système modèle. L'analyse numérique temporelle, validée expérimentalement, associée à l'analyse aux valeurs propres permettent d'étudier de façon complète les instabilités de contact de ce système d'étude.

Le système est instable lorsque le coefficient de frottement est supérieur à 0,12 . L'analyse temporelle montre que le système vibre principalement selon un mode de la structure assemblée qui correspond au mode donné instable à la même fréquence $(2700 \mathrm{~Hz})$ par l'analyse aux valeurs propres. Elle montre également que les conditions locales de contact (forces de contact et vitesses relatives) peuvent être beaucoup plus sévères que celles imposées macroscopiquement.

Les résultats obtenus montrent également que l'analyse aux valeurs propres surestime le nombre de fréquences potentiellement instables. Grâce à l'analyse temporelle, on connaît la fréquence réelle des vibrations du système lors des instabilités de contact.

Les deux analyses menées parallèlement fournissent donc des résultats complémentaires.

- L'analyse temporelle fournit les valeurs de déplacements, vitesses, accélérations et forces de contact en fonction du temps (en régime stable ou instable) permettant de comprendre la dynamique globale et locale de contact du système en fonction du temps. Elle fournit les données d'entrée pour d'éventuelles études acoustiques ou tribologiques.

- L'analyse aux valeurs propres fournit les fréquences propres potentiellement instables. L'observation des modes correspondants montre que la coalescence a lieu entre deux modes dont l'allure est proche et dont l'amplitude modale de déplacement au niveau du contact est importante. Ce type d'analyse permet d'avoir des résultats sur la propension au crissement avec des temps de calculs faibles.

Les analyses aux valeurs propres et temporelle menées en parallèle sur un système permettent d'étudier complètement son crissement éventuel. Pour des systèmes simples ou des systèmes plus complexes, la mise en place des deux analyses permet de disposer de tous les outils pour étudier le comportement du système, l'incidence des vibrations sur le bruit et l'usure et de repousser le crissement à des coefficients de frottement plus élevés en modifiant des paramètres du système (géométrie, matériau).

\section{Références}

[1] R.A. Ibrahim, Friction-induced vibration, chatter, squeal, and chaos, Part I: Mechanics of contact and friction, ASME Appl. Mech. Rev. 47 (1994) 209-226

[2] R.A. Ibrahim, Friction-induced vibration, chatter, squeal, and chaos, Part II: Dynamics and modeling, ASME Appl. Mech. Rev. 47 (1994) 227-253

[3] A. Akay, Acoustic of friction, Acoustic Soc. Am. (2002) $1525-1548$

[4] P. Duffour, J. Woodhouse, Instability of systems with a frictional point contact. Part 1: basic modelling, J. Sound Vib. 271 (2004) 365-390

[5] H.R. Mills, Brake squeal, Institution of Automobile Engineers, 1938 
[6] H. Block, Fundamental mechanical aspect of boundary lubrication, SAE J. 46 (1940) 54-68

[7] R.T. Spurr, A theory of brake squeal, Proceedings of the Automotive division, Inst. Mech. Eng. 1 (1961) 33-52

[8] M. North, Disc brake squeal, Proceeding of the Institution of Mechanical Engineering C38/76, 1976 pp. $196-176$

[9] J.E. Mottershead, S.N. Chan, Brake squeal - an analysis of symmetry and flutter instability, Friction-induced vibration, chatter, squeal and chaos, ASME, 49 (1992)

[10] S.W. Kung, K.B. Dunlap, R.S. Ballinger, Complex eigenvalue analysis for reducing low frequency Brake squeal, Soc. Automotive Eng. 109 (2001) 91-123

[11] W. Hendricx, F. Garesci, A. Pezzutto, H. Van der Auweraer, Experimental and numerical modelling of friction induced noise in disc brakes, SAE Int. 1 (2002) 1566-1571

[12] J. Flint, J. Hultén, Lining-deformation-induced modal coupling as squeal generator in a distributed parameter disc brake model, J. Sound Vib. 254 (2002) 1-21

[13] J. Huang, C.M. Krousgrill, A.K. Bajaj, Modeling of automotive drum brakes for squeal and parameter sensitivity analysis, J. Sound Vib. 289 (2006) 245-263

[14] A. Tuchinda, N.P. Hoffmann, D.J. Ewins, W. Keiper, Effect of pin finite width on instability study of the pinon-disc system, Proceedings of the International modal analysis conference 1, 2002, pp. 552-557

[15] L. Baillet, S. D'Errico, Y. Berthier, Influence of sliding contact local dynamics on global friction coefficient variation, Revue Européenne des Éléments Finis 14 (2005) 305-322

[16] V.G. Oanca, T.A. Laursen, Dynamics of a state variable frictional law in finite element analysis, finite elements in analysis and design, Elsevier, 1996 pp. 25-40

[17] V.G. Oanca, T.A. Laursen, Stability analysis of state dependent dynamic frictionnal sliding, Int. J. Non-Linear Mechanics 32 (1997) 837-853

[18] H. Ouyang, J.E. Mottershead, D. J. Brookfield, S. James, M. P. Cartmell, A methodology for the determination of dynamic instabilities in a car disc brake, Int. J. Vehicle Design, Special Issues on Brake Roughness, Noise, Vibration and Dynamics 23 (2000) 241-262

[19] J. J. Sinou, F. Thouverez, L. Jezequel, Non-linear stability analysis of a complex rotor/stator contact system, J. Sound Vib. 278 (2004) 1095-1129

[20] M.L. Chargin, L.W. Dunne, D.N. Herting, Nonlinear dynamics of brake squeal, Finite element in analysis and design 28 (1997) 69-82
[21] Y.S. Lee, P.C. Brooks, D.C. Barton, D.C. Crolla, A predictive tool to evaluate disc brake squeal propensity, Part 1: the model philosophy and the contact problem, Int. J. Vehicle design 31 (2003) 289-308

[22] Y.S. Lee, P.C. Brooks, D.C. Barton, D.C. Crolla, A predictive Tool to evaluate disc brake squeal propensity, Part 2: system linearization and modal analysis, Int. J. Vehicle design 31 (2003) 309-329

[23] Y.S. Lee, P.C. Brooks, D.C. Barton, D.C. Crolla, A predictive tool to evaluate disc brake squeal propensity, Part 3: parametric design studies, Int. J. Vehicle design 31 (2003) 330-353

[24] D. Vola, M. Raous, J.A.C. Martins, Friction and instability of steadysliding: squeal of a rubber/glass contact, Int. J. Num. Methods Eng. 46 (1999) 1699-1720

[25] A.R. Abu Bakar, H. Ouyang, Complex eigenvalue analysis and dynamic transient analysis in predicting disc brake squeal, Int. J. Vehicle Noise and Vibrations 2 (2006) $143-156$

[26] F. Massi, L. Baillet, O. Giannini, A. Sestieri, Brake squeal: linear and nonlinear numerical approaches, accepted in Mechanical Systems and Signal Processing (2007)

[27] N.J. Carpenter, R.L. Taylor, M.G. Kantona, Lagrange contrainsts for transient finite element surface contact, Int. J. Numerical Methods Engineering 32 (1991) 103128

[28] A. Cochard, J.R. Rice, Fault rupture between dissimilar material: Illposedess, regularization and slip-pulse response, J. Geophysical Res. 105 (2000) 891-907

[29] V. Prakash, R. J. Clifton, Time resolved dynamic friction measurements in pressure-shear, Experimental Techniques in the dynamics of deformable Solids, (AMDVol. 165), ASME, New-York, 1993, pp. 33-48

[30] A. Meziane, L. Baillet, B. Laulagnet, Instabilités de frottement : approches temporelle et fréquentielle, Proceedings of Colloque de Vibrations, Chocs et Bruit, Lyon, France, 2006

[31] A. Meziane, S. D'Ericco, L. Baillet, B. Laulagnet, Instabilities generated by friction in a pad-disc system during the braking process, Tribology Int. 40 (2007) 11271136

[32] Q.S. Nguyen, Instability and friction, Compte rendus de Mécanique 331 (2003) 99-112

[33] D. Guan, J. Huang, Study on brake squeal by feed-in energy analysis, SAE (2001) 69-72 